\title{
Androgen deprivation therapy for metastatic salivary gland cancer
}

\author{
Arielle Elkrief MD, Ramy Saleh MD MSc
}

Cite as: CMAJ 2018 August 20;190:E985-7. doi: 10.1503/cmaj.180286

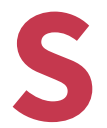

alivary gland cancer is an uncommon cancer that accounts for $0.5 \%$ of all malignancies and $7 \%$ of head and neck cancers. The incidence of salivary gland cancer is increasing and the mortality rate has not decreased. This group of tumours is heterogeneous in terms of location, histology and prognosis. Increasing age is the most important risk factor for salivary gland cancer. Most patients with salivary gland cancer are given a diagnosis of metastatic disease on presentation. The most common site of distant metastasis is the lung. ${ }^{1}$

Because of the rarity of this disease, there are no adequate randomized controlled data that outline the best approach for management. The mainstay of treatment in localized disease is surgical resection. A Canadian study found that older adults represented $40 \%$ of patients with salivary gland cancers. ${ }^{2}$ However, these patients were less likely to undergo surgical resection owing to their comorbidities. ${ }^{2}$ Patients who are not candidates for surgical resection receive radiation therapy, and the 10-year overall survival is between $15 \%$ and $25 \% .{ }^{3}$ It has been postulated that salivary duct carcinoma is histologically similar to prostate cancer, given androgen receptor overexpression in $40 \%-93 \%$ of cases. ${ }^{4}$ Several case reports ${ }^{1,3-5}$ and one case series ${ }^{6}$ have shown that androgen deprivation therapy can be used safely and effectively in patients with metastatic salivary gland cancer.

Box 1 describes a case in which we used androgen deprivation therapy with apparent success. The response was dramatic, albeit not complete. The patient went from having symptomatic pleural effusions and having an Eastern Cooperative Oncology Group (ECOG) performance status of 3 to no longer needing his pleural drains and an ECOG of 0 . We were able to control his disease and maintain a good quality of life; therefore the term "success." There were still brain lesions, but these had stopped growing and the patient was no longer symptomatic.

\section{What is androgen deprivation therapy?}

Androgen deprivation therapy is widely used in the standard treatment of advanced prostate cancer. Luteinizing hormonereleasing hormone analogues are used to suppress the production of androgens in the testes. In addition to a luteinizing hormone-releasing hormone analogue, a direct androgen antagonist is added to the regimen to suppress the effects of

\section{KEY POINTS}

- There are no standardized treatments for unresectable and metastatic salivary gland cancer.

- Salivary gland cancer has been shown to be histologically similar to prostate cancer and widely positive for androgen receptor expression, making androgen deprivation therapy a promising therapeutic option.

- Several case reports and one case series have shown the effectiveness of androgen deprivation therapy in metastatic or unresectable salivary gland cancer, but more data are needed before this therapy can be widely recommended.

androgens in the adrenal glands. These direct androgen antagonists inhibit the binding of dihydrotestosterone to the androgen receptor. Bicalutamide is the most well-studied direct androgen antagonist ${ }^{5}$ and has the most favourable adverse effect profile. Bicalutamide is usually prescribed orally as $150 \mathrm{mg} / \mathrm{d}$ and continued until disease progression. ${ }^{5}$

Several case reports have reported bicalutamide's safety and efficacy in patients with metastatic salivary gland tumours (Box 2). The patients described in these reports were not clearly symptomatic from their disease, and only one patient had brain metastases. Our patient had marked impairment in his quality of life secondary to metastatic pleural effusions and brain metastases. After androgen deprivation therapy, he no longer required the insertion of a tunnelled pleural catheter for pleural effusions and he was able to resume functioning.

\section{Who is eligible?}

In patients who have recurrent or metastatic or unresectable, locally advanced androgen receptor-positive salivary gland cancers despite standard of care therapy, androgen deprivation therapy may be considered. There are no absolute contraindications to this treatment. ${ }^{6}$

\section{What are the harms?}

Although there are no absolute contraindications, androgen deprivation therapy may cause adverse effects that can affect the 


\section{Box 1: Case description}

A 72-year-old man presented to the oncology clinic for management of his metastatic salivary gland cancer. The patient was initially treated with total parotidectomy, followed by adjuvant chemotherapy with weekly carboplatin and paclitaxel, as well as radiation therapy. On routine follow-up computed tomography scan three weeks after completion of treatment, the patient was found to have mediastinal lymphadenopathy. He underwent active surveillance for two years and was stable until he developed progressive general deterioration with a decline in performance status to Eastern Cooperative Oncology Group (ECOG) 3. Computed tomography scan of his chest revealed progression of mediastinal lymphadenopathy, new multiple bilateral spiculated lung nodules and a moderately sized pericardial effusion. The patient had also developed interval new bilateral pleural effusions that required tunnelled pleural catheter placement and intermittent drainage. A magnetic resonance imaging scan of the brain revealed a focal-enhancing $18 \mathrm{~mm}$ lesion in the inferior aspect of the right cerebellar hemisphere compatible with a leptomeningeal metastasis, as well as a second $6 \mathrm{~mm}$ lesion in the upper right cerebellar hemisphere. The patient underwent endobronchial ultrasound-guided biopsy of a mediastinal lymph node, and the pathology revealed metastatic adenocarcinoma with morphology and immunophenotype compatible with salivary duct carcinoma. Androgen receptor was tested and was positive.

Given the lack of available standard therapeutic options, androgen deprivation therapy was recommended and the patient was treated with goserelin (a luteinizing hormone-releasing hormone agonist) and bicalutamide. Within two weeks of therapy, the patient no longer required intermittent drainage of his pleural effusion and the catheters were therefore removed. His performance status improved to ECOG 0 . Computed tomography scan after two months of androgen deprivation therapy showed resolution of the pleural effusions and pericardial effusions, as well as a decrease in size of the pulmonary nodules $(7 \times 4 \mathrm{~mm}$ nodule that had previously measured $8 \times 5 \mathrm{~mm}$, as well as a $3 \mathrm{~mm}$ nodule that had previously measured $7 \mathrm{~mm}$ in the right upper lobe), and near complete resolution of left upper lobe pulmonary nodules. Magnetic resonance imaging of the brain showed stability of the two brain lesions. patient's quality of life. The most common adverse effects include vasomotor symptoms, such as hot flashes with nausea. Gynecomastia is also common, but rarely leads to discontinuation of therapy. Sexual dysfunction occurs frequently, with a loss of libido preceding the onset of erectile dysfunction. The risk of osteoporosis and bone fractures is also increased in androgen deprivation therapy. ${ }^{7}$

\section{What is the evidence so far?}

There are currently no standardized therapies for recurrent, unresectable or metastatic salivary gland cancers. Five-year survival for metastatic salivary gland cancer is $39 \% .^{8}$ The added benefit of chemotherapy and radiation is unclear and grade 3-4 toxicity on the Common Toxicity Criteria scale occurs frequently. ${ }^{9}$ Androgen deprivation therapy was shown to be beneficial and safe in several case reports and a case series of 10 patients. The case series showed clinical benefit in five patients, with a partial response in two patients and stable disease in three patients. The median progression-free survival of these patients was 12 months. ${ }^{6}$ A prospective phase II study of 36 patients with metastatic or unresectable salivary gland cancer showed that androgen deprivation therapy was effective with a median progression-free survival of 8.8 months and a median overall survival of 30.5 months. ${ }^{10}$

\section{What can we expect in the future?}

Although a prospective randomized clinical trial would be the optimal study design to determine the efficacy of androgen deprivation therapy compared with systemic chemotherapy, the low incidence of these tumours make this challenging. Alternatively, collaboration between multiple oncological centres to identify patients with a biopsy-proven androgen-receptorpositive tumour, who had previously failed standard systemic therapy, may facilitate correlation between androgen-receptor mutation status and tumour response using tissue samples.

Box 2: Characteristics of cases of salivary duct adenocarcinoma treated with androgen deprivation therapy

\begin{tabular}{|c|c|c|c|c|}
\hline Author & No. of cases & Age, yr (median) & Androgen deprivation therapy & Response \\
\hline Locati et al. ${ }^{6}$ & 7 & 68 & Bicalutamide + LHRH analogue & $\begin{array}{l}1 \mathrm{CR} \\
2 \mathrm{PR} \\
1 \mathrm{SD}\end{array}$ \\
\hline Jaspers et al. ${ }^{5}$ & 10 & 66 & 9 bicalutamide, 1 bicalutamide + LHRH analogue & $\begin{array}{l}2 P R \\
3 \mathrm{SD} \\
5 \mathrm{PD}\end{array}$ \\
\hline Soper et al. ${ }^{3}$ & 1 & 87 & Bicalutamide + LHRH analogue + RT & $\mathrm{CR}$ \\
\hline Yamamoto et al. ${ }^{11}$ & 1 & 66 & Bicalutamide & CR \\
\hline Agbarya et al. ${ }^{1}$ & 1 & 57 & Bicalutamide & $\mathrm{CR}$ \\
\hline Urban et al. ${ }^{4}$ & 1 & 45 & Abiraterone, prednisone and LHRH analogue & CR \\
\hline Our patient (Box 1) & 1 & 72 & Bicalutamide + LHRH analogue & PR \\
\hline
\end{tabular}


Furthermore, the types of androgen deprivation therapy used in the case reports and case series were heterogeneous, and more studies are required to determine the optimal choice of androgen deprivation therapy.

\section{References}

1. Agbarya A, Billan S, Nasralla $\mathrm{H}$, et al. Hormone dependent metastatic salivary gland carcinoma: a case report. Springerplus 2014;3:363.

2. Eskander A, Irish J, Freeman J, et al. Overview of major salivary gland cancer surgery in Ontario (2003-2010). J Otolaryngol Head Neck Surg 2014;43:50.

3. Soper MS, Iganej S, Thompson L. Definitive treatment of androgen receptorpositive salivary duct carcinoma with androgen deprivation therapy and external beam radiotherapy. Head Neck 2014;36:E4-7.

4. Urban D, Rischin D, Angel C, et al. Abiraterone in metastatic salivary duct carcinoma. J Natl Compr Canc Netw 2015;13:288-90.

5. Jaspers HC, Verbist BM, Schoffelen R, et al. Androgen receptor-positive salivary duct carcinoma: a disease entity with promising new treatment options. J Clin Oncol 2011;29:e473-6.

6. Locati LD, Perrone F, Cortelazzi B, et al. Clinical activity of androgen deprivation therapy in patients with metastatic/relapsed androgen receptor-positive salivary gland cancers. Head Neck 2016;38:724-31.

7. Nguyen PL, Alibhai SM, Basaria S, et al. Adverse effects of androgen deprivation therapy and strategies to mitigate them. Eur Urol 2015;67:825-36.

8. Cancer survival statistics 1992 to 2003. Ottawa: Statistics Canada; 2012. Cat no 82-226-X. Available: www.statcan.gc.ca/pub/82-226-x/82-226-x2012001-eng. htm (accessed 2018 Jan. 3).
9. Laurie SA, Ho A, Fury M, et al. Systemic therapy in the management of metastatic or locally recurrent adenoid cystic carcinoma of the salivary glands: a systematic review. Lancet Oncol 2011;12:815-24.

10. Fushimi $\mathrm{C}$, Tada $\mathrm{Y}$, Takahashi $\mathrm{H}$, et al. A prospective phase II study of combined androgen blockade in patients with androgen receptor-positive metastatic or locally advanced unresectable salivary gland carcinoma. Ann Oncol 2018;28: 979-84.

11. Yamamoto N, Minami S, Fujii M. Clinicopathologic study of salivary duct carcinoma and the efficacy of androgen deprivation therapy. Am J Otolaryngol. 2014;35:731-5.

\section{Competing interests: None declared.}

This article has been peer reviewed.

The authors have obtained patient consent.

Affiliations: Internal Medicine (Saleh), McGill University; Internal Medicine (Elkrief), McGill University Health Centre

Contributors: The original idea for this article was conceived by Ramy Saleh. Arielle Elkrief was responsible for preparing the original manuscript and Ramy Saleh supervised. Both authors revised the manuscript critically for important intellectual content, gave final approval of the version to be published and agreed to be accountable for all aspects of the work.

Correspondence to: Arielle Elkrief, Arielle.Elkrief@mail.mcgill.ca 